Copyright ( 2013 IEEE. Personal use of this material is permitted. Permission from IEEE must be obtained for all other uses, in any current or future media, including reprinting/republishing this material for advertising or promotional purposes, creating new collective works, for resale or redistribution to servers or lists, or reuse of any copyrighted component of this work in other works. 


\title{
Social network Perception Alignment of E-recruiters and Potential Applicants
}

\author{
Torsten Reiners \& Paul Alexander \\ Logistics and Supply Management, Curtin University, Bentley, WA, 6102, Australia \\ t.reiners@curtin.edu.au,p.alexander@cbs.curtin.edu.au
}

\begin{abstract}
Benefits from online social networking are being incorporated into the selection processes used by erecruiters. While this offers great potential for both recruiters and applicants, especially in an increasingly globalized environment, it requires both parties to have mutual understanding of each other's perceptions. This paper empirically explores a global sample containing 1498 applicants from 68 countries and 405 recruiters from 39 countries. We find that both students and recruiters underestimate the impact of each other's social network profile. A model is presented based on communication theory is used to explain these gaps. These gaps in perception will act as barriers to better utilization of global e-recruitment and need to be closed to allow efficient and effective use of social media for this function.
\end{abstract}

\section{Introduction}

The core of the recruitment process is to match the best applicants' resumes with clients' positions. Traditionally this has required a sequential process consisting of developing client position descriptions, advertising the position, acquiring applicant's resumes, manually cross-checking applicants against job requirements, conducting applicant interviews, and making final selections [1].

In the last ten years this process has changed dramatically as online recruitment, commonly termed e-recruitment, has gained rapid acceptance [2]. Erecruitment offers 24 hour access to applicants, clients and recruiters alike. The effective range of applicant searching is much broader, clients can easily access both national and international applicants, and selection of applicants can be supported by filtering and cross-checking online resources. Due to web form standardization the process can be simplified if not automated completely [3, 4]. The recruitment process overall has also been transformed from mainly sequential to parallel. For instance, advertising, applicant acquisition and preliminary filtering are now all concurrent processes for positions placed online in sites such as Seek.com (http://www.seek.com.au) and Monster.com (http://www.monster.com).

Additionally much of the work load has been moved to the applicant, making the recruiting process not only less expensive for the recruiter and client, but more responsive for the applicant. For instance, a potential applicant typically self-selects for positions through "profiles" and fills in web forms constraining to prerequisite selection criteria. Such applications are immediately ready for final screening by recruiters.

Because of its centrality, successful recruiting depends on the effectiveness and efficiency of these online tools and the processes they generate $[5,6]$. From the recruiter's perspective, the aim is to contact as many potentially suitable applicants as possible, to do this better than competitor recruiters and clients, and to process applications fast and cost effectively. For applicants, the aim is to maximize success of selection for the highest number of suitable positions.

It is not surprising that social media, with its ability to provide relationship networks for people with like and complementary interests, is actively used in recruitment processes [7]. For instance, in $201186.6 \%$ of businesses used LinkedIn for recruiting, while 55.3\% used Facebook, and 46.6\% used Twitter [8].

The commonly accepted definition of social networks is given by Boyd and Ellison (2007) as "webbased services that allow individuals to construct a public or semi-public profile within a bounded system, articulate a list of other users with whom they share a connection, and view and traverse their list of connections and those made by others within the system." [9]. Facebook dominates general academic interest [10], however, there are many Social Network Sites (SNS). Some SNS have a more business focus in which users engage in creating a professional profile aimed at benefiting career opportunities [11]. Compared to Facebook, profiles on these social networks focus on presentation of skills and professional background similar to résumés [12].

Recruiters typically use these, not to gain new information (which they obtain primarily from the candidate's résumé) but to validate those résumés and learn about the applicants' network. 
Facebook, while not the leader in use as an aid to recruitment, is nevertheless the globally dominant social network, with grew in just two years from 600 to 900 million interconnected members; with more than 400 million being active at least six out of seven days [13]; facebook.com). The business model is based on exploiting human-to-human and human-to-organisation relationships and the data that flows from these. Social networks promise, because of their sheer size, far broader information base and less guarded information capture regime; to be valuable to e-recruiters.

There is considerable value in understanding how applicants use social networks for recruiting activities, and how recruiters cross-check lodged resumes with profile pages, photos and other information from Facebook. This information clearly offers advantages to the recruiter and client organization since it provides an independent and differently focused source of information about the client, albeit with an overhead in searching and assimilating this information. For the applicant, being in social networks helps provide information that may favourably differentiate them from other (applicants), and may also align them with favourably-perceived social groups or interests. However, this information is not specifically tailored to be useful for this purpose, and often is not even consciously entered (and controlled) by the applicant directly, and therefore introduces significant risks in terms of negatively interpreted information. Clients too, may prefer to have targeted and referenced information to work with, and this is borne out by the higher business use of a specialist recruitment social network such as LinkedIn compared to Facebook.

Facebook and online recruiting are potentially symbiotic, and could therefore be anticipated to evolve in a convergent manner with online applications that merge the two.

The SNS literature in the context of recruiting, especially from the perspective of e-recruiters, is still sparse [10, 14]. Wolfswinkel et al. [15] notes that Anderson [16] was the last to specifically refer to SNS in the context of e-recruiting. Those that do, focus on single countries, which given the globally pervasive nature of this technology, may well represent an artificially biasing constraint.

This study considers reciprocal perceptions of the use of social networks by both parties; that is, how each party perceives the same aspects of social networks features. This is important since what a potential applicant or recruiter places onto social networks, and what they look for on that social networks when searching information about the other, directly affects the way social networks will be used by both parties. Differences in these perceptions will lead to inadequate or inappropriate information being posted as well as incomplete and incorrect searches being conducted; in short leading to an inefficient and ineffective use of this medium.

The attitudes and use of Facebook by recruiters and applicants may be impacted by the context of the home country of both these parties. [17] showed a difference in selection practice by country and this could be imputed to extend to attitudes and use of social networks in this context. For this reason, the study is scoped multi-nationally across 68 countries.

Representation of all applicants covers an enormous demographic, so this study focuses on graduation tertiary students seeking employment, as these represent at least an important subset. A sample of 1498 students is considered across the 68 countries.

To represent recruiters, HR-recruiting decision maker representatives in 405 organisations across 39 countries are considered.

Analysis of this large and diverse body of information is then used to determine perceived use, value and features of social networks from both recruiter and applicants' perspective. Gaps and alignments so determined provide a blueprint for what needs to be reinforced and what needs to be improved in enhancing overall e-procurement effectiveness.

\section{Methodology}

A two factor-alignment of SNS view, perception and importance of profiles of recruiting organisations (recruiters) and students (representing applicants) is tested against the following hypothesis:

HO: Both, students and recruiters underestimate the impact of each others' social network profile

A prior pilot study [18] was conducted with a small group (40 people) in four countries (Germany, Russia, China, USA). The test group finished the survey, but were also interviewed for further insight in the anticipated study. The outcome was used to generate four supporting hypotheses (H1-H4); see also the discussion in Section 5 for more details:

H1. Students underestimate the impact of their activities within SNS upon employers' selection decision

H2. Students and recruiters consider different assessment criteria for hiring

H3. Recruiters misjudge the impact of company social network profiles on students' decisions to apply for a position 
H4. The level of deviation of students' and recruiters' answers is related to the student's country of origin and location of the recruiter's office, respectively.

These hypotheses were tested collectively and individually on a range of representative countries.

\subsection{Experimental Design}

An exploratory study (questionnaire) with a sample of 40 participants in 4 countries [18] was used to generate hypotheses, refine the core survey and set up analyses used for this study. Wherever appropriate this survey considered questions in pairs from both student (S1$\mathrm{S} 13)$ and recruiter (C1-C14) perspective (Figure 1 shows the questionnaire for the later survey based on the experience for the exploratory study). In this paper, specific questions will be referred to using this index.

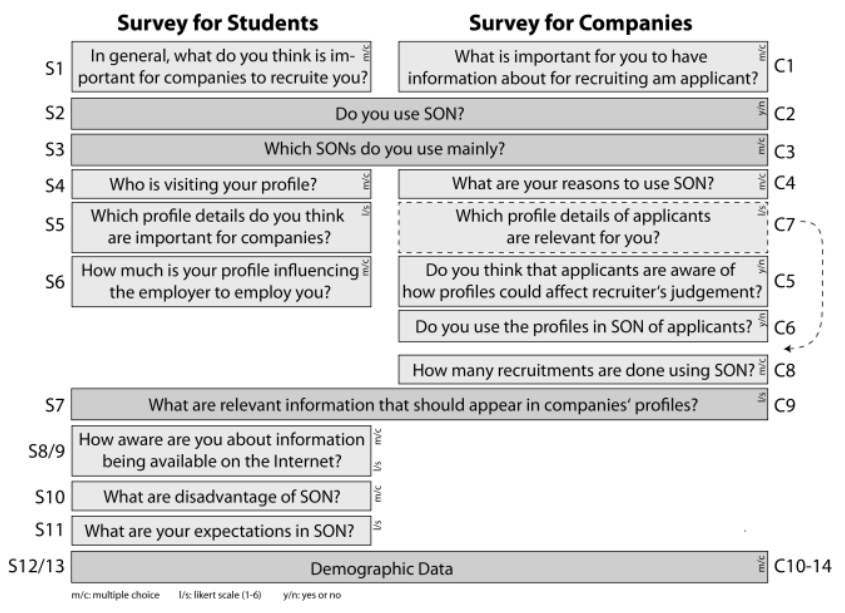

Figure 1: Questionnaire used in the survey

To provide a wide spread of environments in different countries, BRIC countries (Brazil, Russia, India, China) represented very high GDP growth, size (40\% of the world population), and biggest emerging markets. Afghanistan represented a small economy developing country, France a strong and highly developed economy, Poland a fast growing economy, Turkey (tighter censorship) and Asian/European influence, USA as the origin of the largest SNS, and a strong global culture and economy; similar to France (to provide comparison opportunities).

\subsection{Sample}

The survey was conducted mainly online using SurveyShare [19], in several cases by Internet VoIP or phone, or sending the questionnaire to faculties, distributed as paper to students.

To allow meaningful statistical analysis sample size was a minimum of 20 per country. Ten countries were selected for extended analysis based on higher sample responses ( $>50$ students and $>20$ recruiters) to allow more in depth analysis.

Participants were selected from AIESEC (http://aiesec.org). This represents a population of 45,000 students in 107 countries and - through representatives - human resource managers in many companies, who are also accessible for these students. While there is some bias in this sample since AIESEC students have a larger likelihood for a social network affinity, it is argued that the study qualifies for a preliminary study as artefacts (e.g., deviations in students' and recruiters' expectations) would be conservative; that is the average student is assumed to be less aware of effects of their profiles compared to students with more practical experiences.

The data is also skewed towards German students and recruiters due to preferential access (this is the researchers' home country). This is corrected in analyses by using ratios and percentages, and statistically corrected for this effect.

The questionnaire was fully completed by 1497 students from 68 different countries and 405 recruiters from 39 countries, and represents statistically valid samples for the analyses they are subjected to.

\section{Results}

Average age of respondents is 23.35 , a low value expected with the predefined group of participants. The distribution in the sample is 15-19 (9.68\%), 20-24 $(59.8 \%), 25-29(27.59 \%)$, and $>30$ (2.93\%). $57.49 \%$ are female, $42.51 \%$ are male.

India $(66.6 \%)$ and Turkey $(60.2 \%)$ have the highest share of females.

In Afghanistan the proportion of recruiters in small companies ( $<200$ employees) is high, in Germany the recruiters in larger companies ( $>5000$ employee), and in India recruiters in companies with 1001-5000 employees. Otherwise, the size of 201-1000 employees dominates in most countries.

The companies are from different fields; industrial services (21.3\%), financial services (19.26\%) and consultancy $(13.82 \%)$ dominate.

\subsection{Student and recruiter perceptions of recruiting information expectation}

Table 1 shows the importance of recruitment requirements as perceived separately by students and 
recruiters for all countries combined, which aligns with those for Brazil, Turkey, and USA when calculated separately. Variations by country do occur. Some see experience abroad as more important than marks at university (France, Poland, Russia, other), experience abroad over languages (Germany, India), languages over social activity (China), and job experience over ethnical background (Afghanistan; a criteria also important in China).

Table 1: Perceptions of importance of recruitment expectations (all countries collated)

\begin{tabular}{|l|l|l|}
\hline Expectations & Students & Recruiters \\
\hline $\begin{array}{l}\text { Most } \\
\text { important }\end{array}$ & Job experience & Job Experience \\
& $(0.86 \pm 0.13)^{*}$ & $(0.77 \pm 0.15)$ \\
& Languages $(0.70 \pm 0.09)$ & University Marks \\
& University marks & $(0.70 \pm 0.24)$ \\
& $(0.66 \pm 0.21)$ & Language $(0.59 \pm 0.18)$ \\
\hline Least & Hobbies $(0.13 \pm 0.07)$ & Gender $(0.11 \pm 0.09)$ \\
important & Profiles $(0.16 \pm 0.14)$ & Hobbies $(0.14 \pm 0.08)$ \\
& School marks & Ethnic background \\
& $(0.18 \pm 0.14)$ & $(0.25 \pm 0.23)$ \\
& Gender $(0.18 \pm 0.10)$ & \\
\hline
\end{tabular}

* mean and variance shown in brackets here and throughout this paper. Single number in brackets is quoted, this is the mean of the value surveyed.

Some low-importance expectations are ranked more highly in particular countries. Gender is ranked high in Afghanistan, hobbies in China/Turkey, profile in China, marks at schools in Afghanistan and Brazil.
The highest ranked responses of recruiters are the same as those of students, and this tracks those in Afghanistan, Brazil, China, Poland. In France, experience abroad is ranked higher than job experience. In Germany social activities prevail over languages, in India ethnical background over languages, in Turkey marks at school over languages, in USA profile over languages and in the "other countries" category, which consists of collated smallersample countries, experience abroad is more highly valued than marks at university.

In China, France, India, and USA recruiters rank profile high, while students expect this only in China. Profiles are of no importance for recruiters in Turkey, Russia, Poland, and Afghanistan. For recruiters, ethnical background is important in India (0.87), between (0.24) and (0.38) in Russia, China, Poland, and Brazil; USA does not consider this and gender at all. In India, marks at school, gender, social activity, and hobbies have no responds from recruiters.

Figure 2 shows the balance between general expectations by students of what recruiters want to see (S1), and by recruiters of what they pay attention to (C1) in the recruiting process; that is, the difference between student and recruiter perception. A negative value $(\Delta$ perception $=$ student's perception - recruiter's perception) indicates that students see less importance in this criterion than recruiters.



Figure 2: Expectation of students what companies want to see (S1) and what companies pay attention to (C1).

\subsection{Participation in social networks}

Due to bias in the sample already highlighted, there is high participation and knowledge of social networks $(0.87 \pm 0.07)$ with Turkey having the lowest participation at $73.08 \%$.
The same picture is given for the recruiter participation $(0.89 \pm 0.6)$. The data source of the AEISIC network might be expected to deliver respondents with higher interest in networking sites in the first place.

The kinds of broadly defined social networks (offering social networking; including YouTube, 
BlogSpot and Twitter for instance) used by students and recruiters is also explored. The results reflect the dominant position of Facebook for students $(0.79 \pm 0.12)$. Only in Brazil, Facebook (0.53) is less popular than Orkut $(0.78)$, whereas all but India and USA have big gaps even to the second most popular social network, which is YouTube and Twitter. In USA, Facebook (0.85), YouTube (0.81), Twitter (0.74) are all popular. In India, LinkedIn, Orkut, Blogspot, YouTube, and Twitter are commonly used (0.66).

LinkedIn (0.28) and Xing (0.19) are the only offered networks for professional profiles, with Xing mainly used in Germany (0.55) and China (0.37), and LinkedIn used in India (0.77), Brazil (0.36), Afghanistan (0.34), and Turkey (0.33). MySpace is still in use, i.e. in China (0.47) and Afghanistan (0.34). In Germany, Poland, and Russia, many students selected other (>0.43), implying the use of other services (like the VZ-service in Germany) which were not further considered in this research.

For recruiters, the usage of social networks is quite different. Facebook is not the most used network $(0.62 \pm 0.3)$ but it is instead LinkedIn $(0.63 \pm 0.22)$, emphasizing its professional character. Xing $(0.45 \pm 0.29)$ and Twitter $(0.45 \pm 0.21)$ complete the top four services. Again, Brazil has its focus on Orkut $(0.77)$ and India is active in most social networks. Finally, recruiters in USA and Turkey use YouTube and MySpace above average. Facebook has almost no market in China and Russia where other social networks like Vkontakte dominate the market. Nevertheless, recruiters use LinkedIn, showing again the professional interest in using social networks.

\subsection{Relationships represented in social networks}

Assessing why students believe recruiters visit profiles is important as it indicates the awareness by them about potential recipients of their posted information (Figure 3). The picture is internationally very homogenous with friends, friends of friends; and colleagues being most expected and government, HR department, and head-hunter least expected.

Students expect people with commercial interests, or strangers to be more often on their profile than any recruitment-related person, with the exception of China, where students believe the government is even more often on their profile than friends of friends.

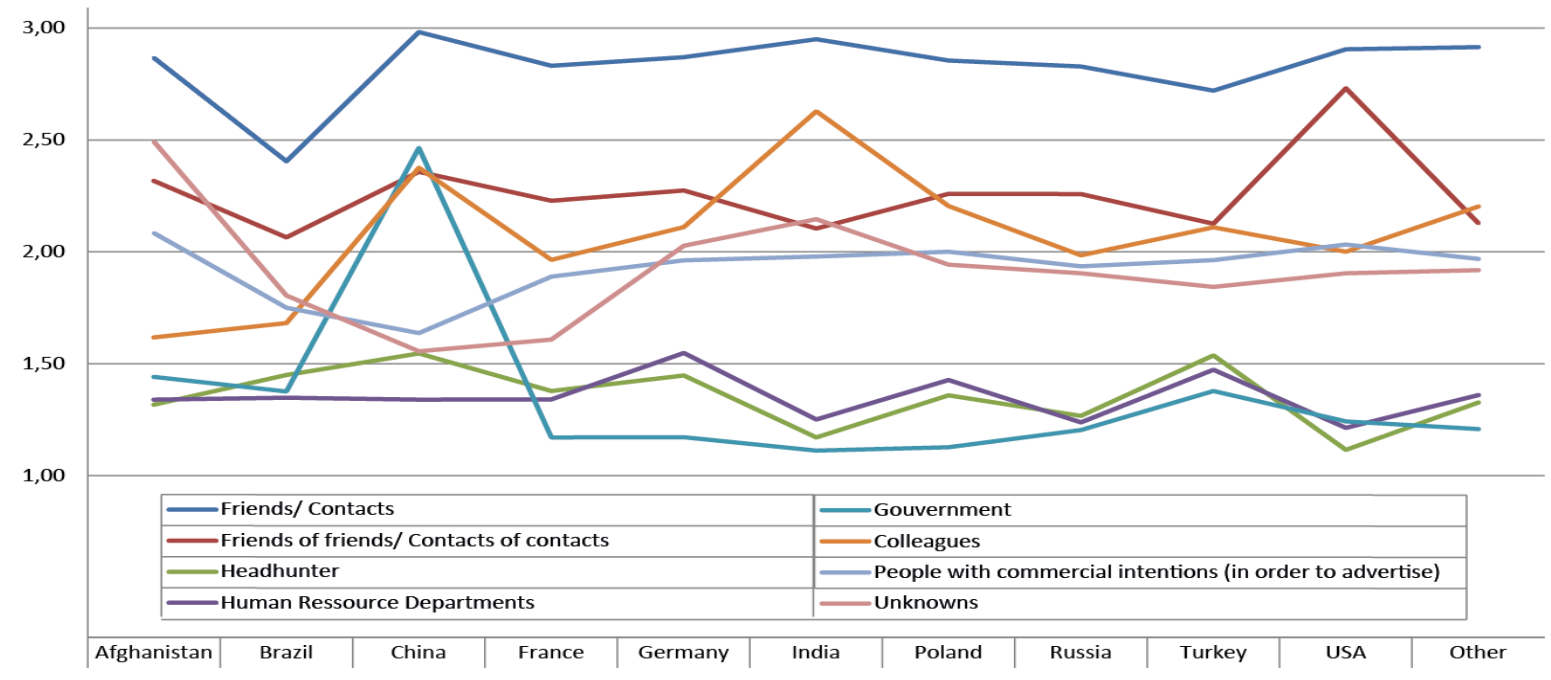

Figure 3: Expected visitors on students' profiles

\subsection{Social network information most important for recruiters}

There are significant differences in the estimates by students and recruiters of what is important profile information. These perceptions were measured on a continuous scale: 1 (not significant) to 5 (critical).
In China, France, Germany, Poland (with the exception of social activities, and age and gender in China and Poland), Russia, and Turkey, students tend to over-estimate the importance of many aspects of their profile.

Afghanistan shows the most deviation as 5 information types have more than a mean of 2 units difference $(\Delta)$ in perception between students and recruiters: gender $(\Delta 2.45)$, qualification $(\Delta 2.26)$, 
appearance $(\Delta 2.85)$, political interest $(\Delta 3.19)$, and nationality $(\Delta 3.7)$. India has 3 information types with more than $\Delta 2$ (qualification, hobbies, group membership).

Brazil is unique among these countries in that the students underestimate the relevance for almost all fields but qualification, with age being the only one " with $\Delta-2.06$ units. In USA 5 information types are overestimated (gender, qualification, hobbies, membership, and nationality) and 7 are underestimated.

For data consolidated for all countries students' overestimate the importance of their profile features in all cases but age $(\Delta-0.13)$, qualification $(\Delta-0.5)$ and job experience $(\Delta-0.42)$.

Gender $(\Delta 0.16)$ and multimedia files $(\Delta 0.46)$ are least important.

\subsection{Influence of social network profiles on employers}

Students' perceptions about the influence a profile might have been generically categorized into "a lot", "slightly" or "not at all". Most students selected "slightly" (0.58), while "a lot" was chosen (0.19) and "not at all" (0.23). Most confident are students in USA (0.78), while all other countries stayed below $(<0.5)$.

"Not at all" and "slightly" were combined into a single category and compared to "a lot". Here, in all countries but Afghanistan and China, "not influencing" dominates. In Afghanistan (0.71) students think that their profile is influencing the employer, in China both opinions are closer together (0.59).

In average, recruiters tend to believe that students are not aware that their profile will be considered during recruitment (0.59). A closer look at the countries reveals that in India (0.96), USA (0.90), China (0.85), France (0.69), Turkey (0.66), Brazil (0.65) and Germany (0.63), most recruiters do not believe that students really know about the possible impacts; which matches the results from question S6. In Afghanistan, recruiters trust students to know about the effects (0.90), same in Poland (0.64) Russia (0.5) undetermined.

\subsection{Recruiters' use of applicants' social network profiles}

Do recruiters look into the profiles of the applicants during recruitment? The answers match the expected visitors on social network sites with one exception. Turkish recruiters using the profile rarely (0.34), while students believe the use is higher.

The amount of recruitment action being undertaken via social networks shows that besides USA (0.55) undertaking $41-50 \%$ of their recruiting with social networks, most have no significant numbers above 30\%. In Afghanistan (0.93) there is no significant use of social network for recruiting, while India is second behind the USA.

\subsection{Reasons for recruiters to use social networks}

In terms of recruiters' main arguments for using social networks (Table 2), besides India, China, and USA, recruiting is not considered a significant reason to use social networking. While India is focusing on recruiting, USA is aiming for a complete integration of social networks in all tasks. All other countries appear to use this medium largely to promote their image and support networking and communication. Russia is different in that recruiters do not use social networks significantly.

Table 2: Reasons for recruiters to use SNS

\begin{tabular}{|c|l|l|l|}
\hline Rank & $\begin{array}{l}\text { Main argument for using social } \\
\text { networks }\end{array}$ & Mean & Variance \\
\hline 1 & expanding business networks & 0.52 & 0.23 \\
\hline 2 & Advertising & 0.44 & 0.24 \\
\hline 3 & Exchanging now-how & 0.44 & 0.24 \\
\hline 4 & Recruiting new personnel & 0.40 & 0.31 \\
\hline
\end{tabular}

The importance of social networks as a new media for communication, exchange of knowhow and advertising is recognizable. Recruiters in developing countries (Afghanistan, China, Turkey, and Brazil) can use the technology to network and gain knowledge.

Overall social networks are not yet replacing emails or other mainstream communication forms but is still used by to chat with friends (0.32), colleagues (0.30), and for email (0.21).

\subsection{Perceptions of what company profiles should include}

Table 3 shows both student and recruiter perceptions of what profiles should contain, as measurements from 1 (not important) to 5 (critical). Some divergences are found for India with respect to earnings and philosophy, for Poland and Russia with respect to career information, and for USA in regard to earnings. 
Table 3: Recruiter and student perceptions of profile requirements

\begin{tabular}{|l|l|l|}
\hline $\begin{array}{l}\text { Profile inclusion } \\
\text { requirements }\end{array}$ & $\begin{array}{l}\text { Student } \\
\text { perception } \\
\text { (mean units) }\end{array}$ & $\begin{array}{l}\text { Recruiter } \\
\text { perception } \\
\text { (mean units) }\end{array}$ \\
\hline job offers & 0.52 & 0.47 \\
\hline $\begin{array}{l}\text { Career enhancement } \\
\text { prospects }\end{array}$ & 0.48 & 0.44 \\
\hline Earnings & 0.47 & 0.40 \\
\hline Company philosophy & 0.43 & 0.40 \\
\hline Reports of employees & 0.42 & 0.36 \\
\hline Appearance of the profile & 0.41 & 0.39 \\
\hline Social activities & 0.39 & 0.38 \\
\hline
\end{tabular}

\subsection{Student perceptions of what is available from their profiles}

Students rated the importance of having availability of their personal data on the internet on a scale of 1 (not aware) to 6 (fully aware). $76.85 \%$ answered with 4-6, uniform for all countries except France, Russia, and Turkey, where more than 38\% rated 1 (Madden et al. 2007). Awareness was further evaluated by the following eight questions (Table 4), which could be ranked from 1 (completely false) to 6 (completely right).

Table 4: Factors of awareness of SNS control

\begin{tabular}{|l|l|}
\hline$(1)$ & $\begin{array}{l}\text { Only a part of the information, which is available about } \\
\text { my personal data on social networks, is provided by me. }\end{array}$ \\
\hline$(2)$ & $\begin{array}{l}\text { Sometimes I am surprised how much information can be } \\
\text { found on social networks about my personal data. }\end{array}$ \\
\hline$(3)$ & $\begin{array}{l}\text { I want to have full control over information about me in } \\
\text { social networks. }\end{array}$ \\
\hline$(4)$ & $\begin{array}{l}\text { I would like to be asked, before any information about me } \\
\text { is published at any web site. }\end{array}$ \\
\hline$(5)$ & $\begin{array}{l}\text { I am scared, that my personal data are used for purposes I } \\
\text { do not know anything about. }\end{array}$ \\
\hline$(6)$ & I worry a lot about my privacy. \\
\hline$(7)$ & I use fake data in my social network profile. \\
\hline$(8)$ & $\begin{array}{l}\text { I retain full control over my information in social } \\
\text { networks. }\end{array}$ \\
\hline
\end{tabular}

Students almost completely agree on (3) $(4.79 \pm 0.88)$ and (4) $(5.05 \pm 0.87)$, while faking information on their profile (7) is not approved of (2.43 \pm 0.79$)$. Confidence about the awareness is shown in the answers to (1) and (2), where all but China gave a score below 3.0 (for 1. 2.95 \pm 1.02 and for 2. $2.88 \pm 0.81$ ).

Question (5) is agreed on by students from Afghanistan, China, France, and Germany, implying that they fear that they are not in charge and the data is passed on to third parties. Besides Germany, students in these countries worry about their privacy. In Afghanistan (4.59) at question (8) contradicts the concerns in questions (5) and (6), as students are worried about their privacy. Brazil $(<3.0)$ is the only country with few worries about where information comes from and who do not necessarily require more control and methods to prevent postings by others.

\subsection{Student Perceptions of the disadvantages of social networks}

Students chose one of 11 predefined disadvantages. The top ranking reasons are shown in Table 5. Interviews with students and recruiters indicate that these perceptions likely result from social networks like Facebook where games and small entertainment apps are dominant and profiles are not work-related but provide fields to describe mainly social activities and interests.

Table 5: Perceptions of disadvantages of social networks

\begin{tabular}{|c|l|l|}
\hline Rank & $\begin{array}{l}\text { Main argument for using social } \\
\text { networks }\end{array}$ & $\begin{array}{l}\text { Percentage } \\
\text { respondents }\end{array}$ \\
\hline 1 & Receiving spam & 64 \\
\hline 2 & Data misuse & 64 \\
\hline 3 & Danger of stalking & 60 \\
\hline 4 & Not taken seriously enough & 36 \\
\hline
\end{tabular}

All other reasons are below 20\% and considered to be not a major concern compared to the others. An international comparison shows that the top four disadvantages are present in almost all countries but serious enough is not an issue in USA, Brazil, China, France, and Germany. The other reasons are below the $20 \%$ line. Afghanistan, Brazil, and Turkey complain about too many members, while Russia is not having enough (insufficient members).

\subsection{Student expectations of the future of social networking}

Students' expectations for the future of social networking were measured on a scale of 1 (unlikely) to 6 (very likely).

Responses were very homogenous over all countries, with only regional variations. For instance, social network cost reductions are not anticipated so highly in India, and students in USA do not see that autonomous data analysis (e.g., automatically linking images to profiles) is likely to come soon.

\section{Analysis}

In order to check the validity of the main hypothesis $\mathrm{H} 0$, the acceptance of the four supporting hypotheses H1-H4 was verified (see [18] for a detailed description of the statistical analysis).

For H1 (applicants underestimate the impact of their activities upon the selection decision) - 
questions $\mathrm{C} 1, \mathrm{C} 4, \mathrm{C} 6, \mathrm{C} 8, \mathrm{~S} 1$, and $\mathrm{S} 4$, we find students do believe that their profile is not visited by head-hunters $(67 \%)$ or HR manager $(64 \%)$ (S4). On the other hand, C4 and C6 show that recruiters use social networks for recruiting (39\%) and check online profiles (42\%). Most recruiters use $1-30 \%$ of their recruiting through social networks. This indicates that applicant underestimate the impact.

For C1 and S1, we applied a Chi Square-test for all items; with the last item about profiles being the most relevant one. The results are $X^{2}=0.039$ and $\varphi=$ 0.047; implying that the items significantly but weakly vary from each other.

According to the results of the other questions, the other items in $\mathrm{C} 1$ and $\mathrm{S} 1$ (see [18] for all $\mathrm{X}^{2}$-tests) we accept $\mathrm{H1}$.

For $\mathrm{H} 2$ (assessment of hiring criteria differs between recruiters and applicants), we use results from $\mathrm{C} 1, \mathrm{C} 7, \mathrm{~S} 1$, and S5. For $\mathrm{C} 1$ and $\mathrm{S} 1$, we used a Chi Square-test which identified 5 items that have a correlation (marks at school, job experience, languages, gender, profile), 2 items fail marginal (social activity, hobbies), and the rest can be assumed coincidently.

For S5 and C7, we used a t-test combined with Cohen's D. The t-test shows a strong correlation for all items but gender, the Cohen's $D$ for 2 items a medium effect (qualification 0.631, nationality 0.517), for 6 a small but strong tendency towards medium effect (social activity 0.496, appearance 0.486 , media on profile 0.45 , groups 0.445 , work experience 0.437 , contacts 0.365$)$, and for 4 an actual small effect $(<0.2)$.

Even though the factual results are not radically different, we can state that the hiring criteria differ. Thus, the hypothesis $\mathbf{H} 2$ is accepted.

For H3 (recruiters misjudge the impact of company social network profiles on applicants' decision to apply for a position), we use the results of question S7 and C9. The t-test shows only for one item a significant correlation (layout of the profile), the Cohen's $D$ effect is small for all items with only three out of seven having a tendency to medium (information about job 0.481, careers changes 0.427 , possible earnings 0.496).

Thus, the hypothesis $\mathbf{H 3}$ cannot be confirmed and is rejected.

For H4 (the levels of divergence between recruiters' and applicants' answers differ, depending on the country of origin, respectively the respondents' branch office), the results of questions C1, C7, C9, S1, S5, and S7 are used. For each matching question (C1/S1, S5/C7, S7/C9), the correlation with the country was examined. As shown in [18], the first two combinations show differences in the answers that can be related to the countries; the last combination (S7/C9) is not showing this relation.

Nevertheless, with two out of three pairs, we found significant differences, such that the hypothesis H4 has to be accepted.

With $\mathrm{H} 1, \mathrm{H} 2$, and $\mathrm{H} 4$ being accepted and demonstrated significant differences in the perception of recruiting in social networks, the main hypothesis H0 (Both, applicants and recruiters, underestimate the impact of each other's social network profile) can be accepted.

\section{Discussion}

This survey shows that the potential of social networks and their impact on both recruiters and applicants is underestimated, and that the differences at the global level are still significant. These international differences in particular are striking and influence what information is posted onto social networks by both parties, and also how it is perceived. For example information posted on a European/US profile is formal and would appear "unfriendly" to Indian recruiters, whereas those posted on Indian profiles might appear amateurish to European recruiters. Within those countries though, alignment of recruiter and applicant perceptions is generally appropriate to serve both party's recruitment objectives.

There are also quality differences in the approach to social networks. Asian profiles tend to include fake information (see Table 3), and this is discounted by recruiters. While this may be appropriate in some countries where a "natural adjustment" is factored in by recruiters, in countries where accuracy of social network profile information is more highly valued (USA, Germany, and other non-Asian countries) Asian recruiters may wrongly devalue the profile information and risk missing candidate opportunities.

Clearly local cultural, political, and social norms govern how social networks are used by all parties, and this stands to reason since they are all built initially from a person's local networks (you connect with your friends, and then their friends and so on), and so this naturally flavours content with what they want to see; a very localized perspective. But erecruitment, while it utilizes those personal networks, is immediately global in focus.

This therefore generates gaps between recruiters and applicants at the global level. This study has highlighted these gaps, which are not just a source of misunderstanding or lost opportunity between recruiter and applicant, but significant barriers to erecruitment's overall efficiency and effectiveness. 
The problems need to be put in the right context however. Social networks are the Internet's expression of the global Zeitgeist and are nothing if not changing. Its users are rapidly evolving different patterns of use. Facebook's rapid replacement of MySpace shows how quickly such reactive changes can occur. It is the users that make these changes, each contributing in a miniscule but nevertheless influential way, like grains of sand in a shifting dune, to the overall form of the medium. Some of these changes are already seen to be rapid; the survey notes countries otherwise similar but at different stages of adoption of social networks that show evolving perceptions driven by expectations of who will be viewing their profiles.

Others, such as safety and security implications in China, may not be so rapid and easy to change. The survey does point to intransigent differences as being an increasingly significant barrier to (global) erecruitment. It will be interesting to observe how social networks and governments balance their needs with global-level business uses that will deliver a benefit to their countries.

How can these findings be explained? The model shown in Figure 4, derived from [20], provides a useful explanatory device. In this model communication is based on a system made of components: an information source, producing a message; a transmitter, encoding the message into signals; a channel transmitting adapted (coded) signals; a receiver decoding the message from the signal; and a destination, where the message arrives.

This model can typically be applied to singledirection communications, in which the message source and destination are fixed (for instance, a bill board or TV commercial), or two-way communications, in which source and destination alternate or operate simultaneously in both modes (for instance, an in-person conversation). In the latter case both recipient and source provide real-time feedback to the other, which refines and directs the communications process aligning both parties' understanding of the communication.

In social networks, there is two-way communication, but there is little real-time feedback. That is, both recruiters and potential applications are transmitting and receiving messages simultaneously and applying static bias's from each party. The recruiter sees an applicant in terms of specific jobalignment suitability and more general perceptions formed from associated social network information. The applicant views the specific job information in terms of the context of the organisation and of his/her own needs and perceptions. Additionally, both make adjustments for cultural/social norms such as degree of truthfulness, privacy concerns and so on. Finally, both parties have their own interpretation of the others' social network presence, derived from differences in demographic and social experiences.

These factors can be seen as "perception filters" which potentially distort the central message, in this case the posted job information.

In a traditional two-way conversation, these filters would be continuously adapted through realtime feedback. In a Social Network, the lack of realtime feedback results in mis-direction and misprioritisation of messages and their importance.

\section{Conclusion}

E-recruitment may be seen as the means to "transform the future of the recruitment industry [by] opening new doors for employers and job seekers to connect in ways not possible before" [21], but this can only be achieved when all parties in all countries know and understand social rules that influence both the content, style and use of their posted profiles.

In research as well as general media, SNS and there usage is considering a general audience, especially if looking into security, perception, application. To our knowledge, strict focus on students and their perception about how it influences their careers (in terms of applying at companies or pursuing an academic path) is not done on a broad level. In addition, we selected a group where we expected a higher perception of their SNS activities with respect to the later; students are in close contact with companies.

This survey confirms that significant gaps exist on this front, and suggests that some distance is yet to be covered. These perceptions must be aligned, particularly in the areas that we have highlighted, in order to allow e-recruitment to expand.

An explanation for these gaps can be found in communication theory, and in particular how social networks reduce real-time feedback while allowing simultaneous two-way communications. In such a communications environment, the contribution of conventions, organisational and social information must be far better understood by both parties prior to (both) using social networks. Adding to the complication is the wide variation of perceptions driven by cultural differences, which in a global recruiting environment, must also be factored in. 




Figure 4: Different perceptions of recruitment-related messages (after [20])

There is much more knowledge needed to make e-recruiting using social networks a reliable and efficient means to connect all the right applicants to all the right jobs and future research will therefore concentrate on strategies for aligning participant messages, and also the importance global factors.

\section{Acknowledgment}

We like to thank Nuno Monteiro and Baktas Dost for their work on the data collection process and the reviewers for their valuable feedback about how to engage in our future research.

\section{References}

[1] S. Taylor and T. Bergmann, "Organisational recruitment activities and applicant's reactions at different stages of the recruitment process," Personnel Psychology, vol. 40, pp. 261-285, 1987.

[2] S. Laumer, A. Eckhardt, and T. Weitzel, "Status Quo and Trends in E-Recruiting - Results from an Empirical Analysis," in Proceedings of the International Conference on Information Resources Management (CONF-IRM), 2009.

[3] R. Compton, W. Morrissey, and A. Nankervis, "Effective Recruitment and Selection Practices," ed: CCH Australia, 2009.

[4] D. Torrington, Laura. H. and S. Taylor, Human Resource Management. Harlow: Pearson Education, 2005.

[5] S. Maurer and Y. Liu, "Developing effective erecruiting websites: Insights for managers from marketers," Business Horizons, vol. 50, pp. 305-314, 2007.

[6] D. Feldman, "Internet Job Hunting: A Field Study Of Applicant Experiences With On-Line Recruiting," Human Resource Management, vol. 41, pp. 175-191, 2002.
[7] K. Shea and K. Wesley, "How social networking sites affect students, career services, and employers," National Association of Colleges and Employers Journal, vol. 66, pp. 26-32, 2006.

[8] Jobvite. (2011). Social Recruiting on the Rise: Jobvite Survey Reveals $89 \%$ of U.S. Recruiters Plan to Use Social Recruiting in 2011. Available: http://recruiting.jobvite.com/news/pressreleases/pr/jobvite-social-recruiting-survey-2011.php

[9] D. Boyd and N. Ellison, "Social Network Sites: Definition, History, and Scholarship," Journal of Computer-Mediated Communication, vol. 13, pp. 210230, 2007.

[10] D. Richter, K. Riemer, and vom Brocke, J., "Internet Social Networking: Research State of the Art and Implications for Enterprise 2.0," Business \& Information Systems Engineering, vol. 2, pp. 89-101, 2011.

[11] D. Thew, "LinkedIn - a user's perspective: using new channels for effective business networking," Business Information Review, vol. 25, pp. 87-90, 2008.

[12] C. Schaefer, "Motivations and usage patterns on social network sites," in Proceedings of the 16th European Conference on Information Systems, 2008, pp. 20882099.

[13] V. Conzensa. (2010). World Map of Social Networks. Available: http://www.vincos.it/world-map-of-socialnetworks

[14] D. H. Kluemper and P. A. Rosen, "Future employment selectionmethods: evaluation social networking web sites," Journal of Managerial Psychology, vol. 24, pp. 567-580, 2009.

[15] J. Wolfswinkel, E. Furtmueller, and C. Wilderom, "Reflecting on e-recruiting research using grounded theory," in Proceedings of the 18th European Conference on Information Systems, 2010, p. 52.

[16] N. Andreson, "Applicant and Recruiter Reactions to New Technology in Selection: A Critical Review and Agenda for Future Research," International Journal of Selection and Assessment, vol. 11, pp. 121-136, 2003.

[17] A. J. Wilkinson, N. A. Bacon, T. Redman, and S. Snell, The SAGE Handbook of Human Resource Management. London: SAGE, 2009.

[18] B. Dost and N. Monteiro, "The (un)aware student: Social Online Networks as a new manner of communication between students and recruiters on the job market," Diploma Thesis, University of Hamburg, Germany, 2010.

[19] Research Tools. (2011). Survey Share: Surveys done simple. Available: www.surveyshare.com

[20] C. E. Shannon and W. Weaver, The mathematical theory of communication. Urbana, Illinois: University of Illinois Press, 1949.

[21] Beyond.com. (2008). Social Networking and its Impact on Online Recruitment. Available: http://www.beyond.com/Media/Online-RecruitmentIn-Social-Networking.asp 